\title{
Principles of Phase Structures in Particle Physics
}




\section{World Scientific Lecture Notes in Physics}

\section{Published}

Vol. 57: Foundations of Quantum Chromodynamics (2nd ed.): An Introduction to Perturbative Methods in Gauge Theories T Muta

Vol. 58: Concepts in Solids, Lectures on the Theory of Solids $P W$ Anderson and $H$ Bunke

Vol. 59: Lattice Gauge Theories: An Introduction (2nd ed.) HJ Rothe

Vol. 60: Massive Neutrinos in Physics and Astrophysics (2nd ed.) R N Mohapatra and $P$ B Pal

Vol. 61: Modern Differential Geometry for Physicists (2nd ed.) $C \mathrm{~J}$ Isham

Vol. 62: ITEP Lectures on Particle Physics and Field Theory (In 2 Volumes) $M$ A Shifman

Vol. 64: Fluctuations and Localization in Mesoscopic Electron Systems $M$ Janssen

Vol. 65: Universal Fluctuations: The Phenomenology of Hadronic Matter $R$ Botet and M Ploszajczak

Vol. 66: Microcanonical Thermodynamics: Phase Transitions in "Small" Systems DHE Gross

Vol. 67: Quantum Scaling in Many-Body Systems M A Continentino

Vol. 69: Deparametrization and Path Integral Quantization of Cosmological Models C Simeone

Vol. 70: Noise Sustained Patterns: Fluctuations \& Nonlinearities Markus Loecher

Vol. 71: The QCD Vacuum, Hadrons and Superdense Matter (2nd ed.) Edward V Shuryak

Vol. 72: Massive Neutrinos in Physics and Astrophysics (3rd ed.) $R$ Mohapatra and $P$ B Pal

Vol. 73: The Elementary Process of Bremsstrahlung W Nakel and E Haug

Vol. 74: Lattice Gauge Theories: An Introduction (3rd ed.) HJ Rothe

Vol. 75: Field Theory: A Path Integral Approach (2nd ed.) A Das

Vol. 76: Effective Field Approach to Phase Transitions and Some Applications to Ferroelectrics (2nd ed.) $J$ A Gonzalo 
World Scientific Lecture Notes in Physics - Vol. 77

Principles of Phase Structures in Particle Physics

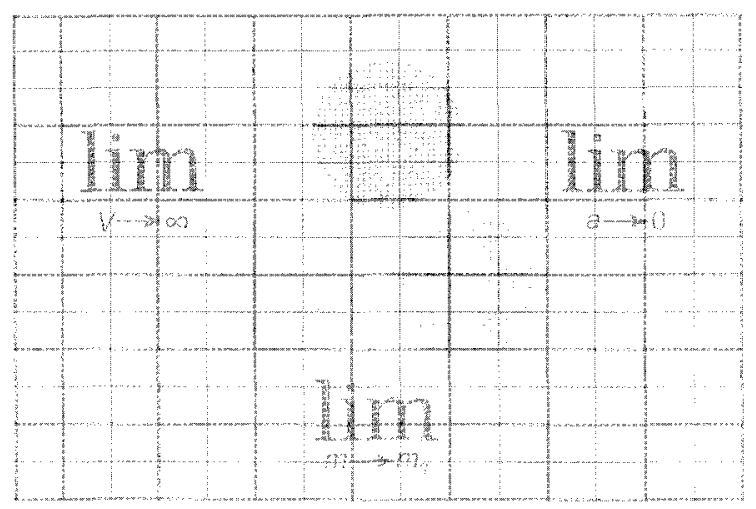

\section{Hildegard Meyer-Ortmanns}

International University Bremen, Germany

\section{Thomas Reisz}

Formerly of Universität Heidelberg, Germany 


\section{Published by}

World Scientific Publishing Co. Pte. Ltd.

5 Toh Tuck Link, Singapore 596224

USA office: 27 Warren Street, Suite 401-402, Hackensack, NJ 07601

UK office: 57 Shelton Street, Covent Garden, London WC2H 9HE

\section{British Library Cataloguing-in-Publication Data}

A catalogue record for this book is available from the British Library.

\section{PRINCIPLES OF PHASE STRUCTURES IN PARTICLE PHYSICS World Scientific Lecture Notes in Physics - Vol. 77}

Copyright (C) 2007 by World Scientific Publishing Co. Pte. Ltd.

All rights reserved. This book, or parts thereof, may not be reproduced in any form or by any means, electronic or mechanical, including photocopying, recording or any information storage and retrieval system now known or to be invented, without written permission from the Publisher.

For photocopying of material in this volume, please pay a copying fee through the Copyright Clearance Center, Inc., 222 Rosewood Drive, Danvers, MA 01923, USA. In this case permission to photocopy is not required from the publisher.

ISBN-13 978-981-02-3441-6

ISBN-10 981-02-3441-4 
To Lara Celine and Anne Sophie (H.M.-O.)

To My Parents (T.R.) 
This page is intentionally left blank

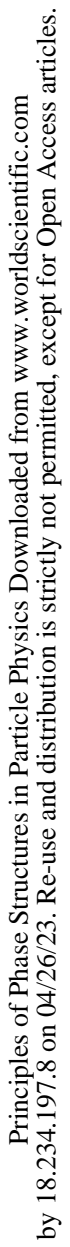




\section{Preface}

Although phase transitions of particle physics appear as exotic phenomena from the viewpoint of daily-life physics, their occurrence in the early universe has far reaching consequences for the status of the universe nowadays and makes these transitions conceptually interesting. "Little bangs" in heavy-ion collisions may reproduce these exotic states of matter in labexperiments, since the available energies in these collisions are meanwhile sufficiently high to produce these states for a very short instant of time. The aim of the book is to derive the phase structure of particle physics by applying standard thermodynamic concepts to particle physics.

The book is based on a review about Phase Transitions in Quantum Chromodynamics by one of the authors (H. M.-O.), published in Rev. Mod. Phys. 68 (1996) 473, and on a series of lectures about Phase Transitions and Critical Phenomena with Applications to Particle Physics at the Universities of Wuppertal and Heidelberg. It was extended to include results on the phase structure of the electroweak part of the standard model, as well as further theoretical developments such as perturbation theory on the lattice at zero and finite temperature, dimensional reduction, flow equations, gap equations and various resummation techniques.

For a detailed understanding of the phase structure of quantum chromodynamics and the electroweak standard model, approximations in analytical and numerical calculations are unavoidable. The aim of the book is to explain these methods and to clearly specify the conditions under which the approximations are under control. The analytical part covers methods both in a continuum regularization and on a spacetime lattice. Since the lattice plays a distinguished role as a nonperturbative treatment of the phase structure of the standard model, it makes up the larger part 
of the book. In the chapter on numerical methods we focus on criteria for an appropriate interpretation of numerical data rather than on details of algorithms to produce them. Where possible we try to bridge the gap between different approaches to one and the same problem, for example between a phenomenological description of first-order phase transitions and rigorous results about their finite-size scaling analysis, or between lattice and continuum approaches, or between effective models and first-principle calculations. The presentation also contains more recent topics which cannot be found in other textbooks so far. To these belong generalized series expansions, dimensional reduction, resummation techniques and certain realizations of the renormalization group.

The book is addressed to graduate and $\mathrm{Ph}$. D. students, to post-docs and interested colleagues, primarily from the field of particle physics. However, to a certain extent it should be of interest for colleagues from the field of statistical physics in general; some tools we are using are not standard ones from statistical physics, but were developed for solving problems in particle physics. Such tools have, for example, applications to the phase structure of spin glasses.

The style of the chapters varies from self-contained, detailed introductions, assuming basic knowledge from undergraduate lectures, to reviews of advanced research. In such reviews we explain the main ideas whose realizations are sometimes quite demanding in the techniques they use. To go there into detail would go beyond the scope of this book. Sections on perturbative approaches can be followed in detail if the reader has expertise in perturbative calculations. In general, we summarize the main ingredients in all chapters to make them self-contained, but the presentation is sometimes condensed so that a basic background knowledge in quantum field theory will facilitate the understanding. On the basis of this book it should be possible to follow the more recent literature on topics in this field.

The aim of the book is neither to give a complete or updated review on possible approaches nor a summary of the state of art nor a complete list of results for the phase structure of particle physics that are available today. The emphasis is on principles.

We are very much indebted to World Scientific for its patience with the submission of our manuscript. For one of us (H.M.-O.) it is also a pleasure to thank the International University Bremen, where it became possible 
to finalize the book during the last years, and in particular to her student Tiberiu Tesileanu for his support in providing a camera-ready manuscript.

Bremen, July 2006

Hildegard Meyer-Ortmanns 
This page is intentionally left blank

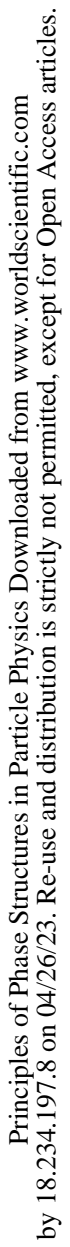




\section{Contents}

Preface vii

1. Introduction 1

2. General Background from Statistical Physics 17

2.1 Generalities . . . . . . . . . . . . . . . 17

2.1.1 Phase transitions in statistical systems . . . . . . 17

2.1.1.1 First- and second-order transitions in the infinite volume limit .......... 17

2.1.1.2 Landau's free energy . . . . . . . . . . 21

2.2 Generating functionals, $n$-point correlations and effective potentials . . . . . . . . . . 26

2.3 The molecular-mean field approximation . . . . . . . . 33

2.3.1 Self-consistent equation of state for a ferromagnet . . 33

2.3.1.1 Critical exponents in the molecular-mean field approximation . . . . . . . . 35

2.3.2 Variational estimates for the free energy of a spin system . . . . . . . . . . . . . . . 41

2.3.3 Molecular-mean field approximation for an $N$-component scalar field theory in $D$ dimensions . . 45

2.3.3.1 Solutions of the mean-field equations .... 50

2.3.3.2 Critical exponents in the symmetric phase . 55

2.3.3.3 Critical exponents in the broken phase . . . 58

2.3.3.4 First-order transitions within the molecular-mean field approximation . . . . . 61

2.3.3.5 Tricritical behavior . . . . . . . . . . 61 
2.3.4 Variational estimates for the $\mathrm{SU}(2)$ Higgs model . . . 62

2.3.4.1 Solutions of the mean-field equations of the $S U(2)$ Higgs model . . . . . . . . . 71

2.3.5 Improved variational estimates for the $S U(2)$ Higgs model . . . . . . . . . . . . . . . . . 74

2.3 .6 Summary . . . . . . . . . . . . . . . . . 79

2.4 Renormalization group . . . . . . . . . . . . . 80

2.4 .1 Generalities . . . . . . . . . . . . . . 80

2.4.2 Block-spin transformations . . . . . . . . . . . 82

2.4.3 Iteration of the block-spin transformation . . . . . 92

2.4 .4 Field renormalization . . . . . . . . . . . . 95

2.4.5 Linearized renormalization-group transformation and universality . . . . . . . . . . . . . . 101

2.4.6 Scaling-sum rules . . . . . . . . . . . . . . . . 104

2.4.6.1 Anomalous dimension . . . . . . . . . . 106

2.4.6.2 Correlation length . . . . . . . . . . 106

2.4.6.3 Two-point susceptibility . . . . . . . . . 108

2.4.6.4 Vacuum expectation value . . . . . . . 108

2.4.6.5 Specific heat . . . . . . . . . . . . . . 109

2.4.6.6 Summary . . . . . . . . . . . 110

2.4.7 Violation of scaling-sum rules for critical exponents . 110

2.5 Finite-size scaling analysis for second-order phase transitions . . . . . . . . . . . . . . . 113

2.5.1 Shift in the pseudo-critical parameter . . . . . . . 118

2.5.2 $\tau$-like and $h$-like scaling fields . . . . . . . 120

2.6 Finite-size scaling analysis for first-order phase transitions . 122

2.6.1 Predictions for rounding and shifting of singularities in first-order transitions . . . . . . . . 129

2.6.2 Finite-size scaling with linked cluster expansions . . 141

2.6.3 Summary of criteria . . . . . . . . . . . 147

3. Field Theoretical Framework for Models in Particle Physics 153

3.1 The standard model in limiting cases I: Spin models as a guideline for the phase structure of QCD . . . . . . 153

3.1.1 Renormalization-group analysis in the chiral limit . . 155

3.1.1.1 Renormalization-group equation, scaling behavior of the Green functions and zeros of the $\beta$-function. . . . . . . . . 158 
3.1.1.2 Computation of the $\beta$-function for an $O(N)$-symmetric scalar field theory . . . 163

3.1.1.3 Generalization to the $S U\left(N_{f}\right) \times S U\left(N_{f}\right)$ symmetric model . . . . . . . . . 166

3.1.1.4 Including nonzero bare masses . . . . . . . 169

3.1.2 Limit of the pure $S U\left(N_{c}\right)$ gauge theory . . . . . . 170

3.1.2.1 Effective versus physical temperatures ... 173

3.1.2.2 The phase structure of $Z(N)$-spin models . 174

3.1.2.3 Influence of dynamical quarks . . . . . . 175

3.1.2.4 Finite quark masses and external fields . . . 176

3.1 .2 .5 Summary . . . . . . . . . . . . . . 178

3.1.2.6 The Columbia plot . . . . . . . . . . 180

3.2 The standard model in limiting cases II: Phase transitions in the electroweak part of the standard model . . . . . . . 183

3.2.1 The electroweak phase transition in cosmology . . . . 183

3.2.2 Perturbative approach in four dimensions in the continuum . . . . . . . . . . . . . 185

3.2.3 The lattice approach in four dimensions . . . . . . 187

3.2.3.1 Gross phase structure with deconfinement and Higgs transition . . . . . . . . . 188

3.2.3.2 The Higgs transition in four dimensions . . 190

3.2.3.3 Effect of the gauge fields . . . . . . . . . 192

3.2.4 The lattice approach in three dimensions . . . . . . 194

3.2.4.1 Measurements of the surface tension . . . . 197

3.2.4.2 Localization of the critical endpoint . . . . 199

3.2.4.3 Universality class of the critical endpoint . . 201

3.2.5 Summary of results and open questions . . . . . . . 202

3.3 A primer to lattice gauge theory . . . . . . . . . . . 204

3.3.1 The QCD Lagarngian . . . . . . . . . . . . . 204

3.3.2 Introducing the lattice cutoff . . . . . . . . 206

3.3.3 Scalar field theories on the lattice . . . . . . . . . . 207

3.3.4 Gauge field theories on the lattice . . . . . . . . . . 212

3.3.4.1 Functional measure and the gauge orbit . . 214

3.3.5 Fermions on the lattice . . . . . . . . . . 216

3.3.5.1 The Wilson-fermion action and hopping parameters . . . . . . . . . . . . 219

3.3.5.2 Staggered fermions and flavor symmetries . 221

3.3.5.3 Sources of errors. . . . . . . . . . . . . . 223

3.3.6 Translating lattice results to continuum physics . . . 224 
3.3.6.1 The critical temperature $T_{c} \ldots \ldots \ldots 226$

3.3.6.2 Choosing the appropriate extension in time direction . . . . . . . . . . 227

3.3.6.3 A test of asymptotic scaling . . . . . . . 227

3.3.6.4 Translation to physical units . . . . . . . . 228

3.3.7 Summary and outlook . . . . . . . . . . . . . 229

3.3.8 Transfer matrix and Polyakov loops . . . . . . . . . 230

3.3.8.1 Path integral formulation of finite temperature field theory . . . . . . . . 230

3.3.8.2 Quantum mechanics of gluons . . . . . . . 233

3.3.8.3 Finite-temperature partition function . . . . 239

3.3.8.4 Representation as path integral . . . . . . 240

4. Analytic Methods on the Lattice and in the Continuum 245

4.1 Convergent versus asymptotic expansions . . . . . . 2 245

4.1 .1 Asymptotic expansions . . . . . . . . . . . . . 247

4.1 .2 Borel resummations . . . . . . . . . . . . . . . . 251

4.1 .3 Polymer expansions . . . . . . . . . . . . . . 252

4.1.4 Strong coupling expansions . . . . . . . . . . 261

4.1.5 A theorem by Osterwalder and Seiler . . . . . . . . 266

4.1.6 Linked cluster expansions as convergent expansions . 273

4.1.7 Convergent power series and the critical region: radius of convergence and physical singularity . . . . 278

4.2 Linked cluster expansions in more detail . . . . . . . . . 284

4.2 .1 Historical remarks . . . . . . . . . . . . . . . . 284

4.2.2 Introduction to linked cluster expansions . . . . . . 287

4.2 .3 Classification of graphs . . . . . . . . . . . . . . 295

4.2.4 Towards a computer implementation of graphs . . . 300

4.2.5 Application to phase transitions and critical phenomena . . . . . . . . . . . 303

4.2 .6 Some results . . . . . . . . . . . . . . . . . . 308

4.3 Renormalization, perturbation theory and universality at zero temperature - the continuum limit . . . . . . . 308

4.3 .1 Generalities . . . . . . . . . . . . . . . 308

4.3.2 Renormalization. . . . . . . . . . . . . 310

4.3.3 Perturbative renormalization . . . . . . . . . 315

4.3.4 The counterterm approach . . . . . . . . . 317

4.3.5 Power counting in the continuum . . . . . . . . 321 
4.3.6 Power counting for Feynman integrals in momentum space: UV-divergence for rational functions and integrals . . . . . . . . . . 322

4.3.7 Power counting for Feynman diagrams:

UV-divergence degrees of propagators, vertices, fields and diagrams . . . . . . . . . . . . . 324

4.3.8 Renormalization or: Removing UV-divergencies under the integral sign . . . . . . . . . . . 326

4.3.9 Renormalization or: Removing UV-divergencies in the counterterm approach . . . . . . . . . 328

4.3.10 Power counting in lattice field theory . . . . . . . . . 329

4.3.11 Preliminaries of lattice-power counting and lattice UV-divergence degrees . . . . . . . . . . . . 331

4.3.12 Power-counting theorem on the lattice . . . . . . 334

4.3.13 Renormalization on the lattice . . . . . . . . . 336

4.3.14 Massless fields and IR-power counting . . . . . . 339

4.3 .15 Gauge theories . . . . . . . . . . . . . . 347

4.4 Weak coupling expansion at finite temperature . . . . . 354

4.4.1 Motivation and problems . . . . . . . . . . . . 354

4.4.2 Finite-temperature Feynman rules and renormalization . . . . . . . . . . . . 358

4.4.3 IR-divergencies and resummation techniques . . . . . 364

4.4.4 Resummation leading to a reasonable weak coupling expansion . . . . . . . . . . . 371

4.4.5 Gauge theories and the magnetic-mass problem . . . 373

4.4.6 Hard-thermal loop resummation . . . . . . . . . 378

4.4.6.1 Example of a hard-thermal loop . . . . . . . 381

4.4.6.2 Effective HTL-action . . . . . . . . . 386

4.5 Constraint effective potential and gap equations . . . . . 388

4.5 .1 Generalities . . . . . . . . . . . . . . . 388

4.5.2 Scalar field theories . . . . . . . . . . . . 390

4.5.2.1 Mass resummation and gap equations . . . . 397

4.5.3 The order of the phase transition . . . . . . . . . 404

4.5.4 The weak-electroweak phase transition . . . . . . 408

4.6 Dimensional reduction at high temperature . . . . . . . . 417

4.6 .1 Generalities . . . . . . . . . . . . . . . 417

4.6.2 Matsubara decomposition and the

Appelquist-Carazzone decoupling theorem . . . . . . 421

4.6.3 General outline of dimensional reduction . . . . . . 429 
4.6.4 Dimensional reduction of a $\Phi^{4}$-theory from four to three dimensions . . . . . . . . . . . 435

4.6.4.1 Large-cutoff and high-temperature expansions of one-loop integrals . . . . . . 442

4.6.4.2 The steps after dimensional reduction . . . . 445

4.6.5 Pure gauge theories and QCD . . . . . . . . . . 448

4.6.5.1 Pure gauge theories . . . . . . . . . . . . . 449

4.6.5.2 QCD with fermions . . . . . . . . . 450

4.6.5.3 Dimensional reduction for pure $S U\left(N_{c}\right)$ gauge theories . . . . . . . . . 454

4.6.5.4 Renormalization and zero-momentum projection . . . . . . . . . . 457

4.6.5.5 Nonperturbative verification of dimensional reduction and determination of the screening masses . . . . . . . . . . . . 465

4.6.5.6 Dimensional reduction in QCD with dynamical fermions . . . . . . . . . . . 469

4.6.6 The Gross-Neveu model in three dimensions . . . . . 477 4.6.6.1 Large $N \ldots \ldots \ldots \ldots$. . . . . . . 482

4.6.6.2 Phase structure at infinite N . . . . . . . . 491

4.6.6.3 Dimensional reduction for finite N . . . . . 493

4.6.6.4 Phase structure at finite $\mathrm{N} \ldots \ldots$. . . . . 500

4.6.6.5 The strong-coupling limit . . . . . . . . . 503

4.6.6.6 Finite couplings and LCE-expansions . . . . 504 4.6.6.7 Summary . . . . . . . . . . . . 506

4.6.7 Dimensional reduction in the $S U(2)$ Higgs model . . 508 4.6.7.1 Guidelines for an alternative form of dimensional reduction . . . . . . . . . . 509

4.6.7.2 Performing the integration step . . . . . 510

4.6.7.3 Integrating upon the superheavy modes . . 511

4.6.7.4 Examples for the matching procedure to integrate upon the superheavy modes . . . . 514

4.6.7.5 Integrating upon the heavy modes . . . . . . 515

4.7 Flow equations of Polchinski. . . . . . . . . . . 517

4.7 .1 Generalities . . . . . . . . . . . . . . . 517

4.7.2 Flow equations for effective interactions . . . . . . 519

4.7.3 Effective average action . . . . . . . . . . 526

4.7.4 High-temperature phase transition of $O(N)$ models . 529

4.7.5 Appendix: Perturbative renormalization . . . . . . 544 
5. Numerical Methods in Lattice Field Theories

5.1 Algorithms for numerical simulations in lattice field theories . . . . . . . . . . . . . . . 549

5.2 Pitfalls on the lattice . . . . . . . . . . . . . 554

5.3 Pure gauge theory: The order of the $S U(3)$-deconfinement

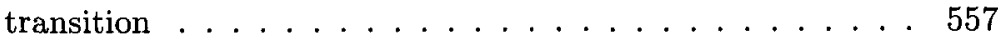

5.3 .1 The order of limits . . . . . . . . . . . 557

5.3.2 Correlation lengths, mass gaps and tunneling events . . . . . . . . . . . . . 558

5.3.3 Correlation functions in the pure $S U(3)$ gauge theory . . . . . . . . . . . 560

5.4 Including dynamical fermions . . . . . . . . . . 561

5.4 .1 Finite-size scaling analysis . . . . . . . . . . . 562

5.4 .2 Finite-mass scaling analysis . . . . . . . . . . 563

5.4 .3 Bulk transitions . . . . . . . . . . . . . . . . 568

5.4.4 Results for two and three flavors: The physical mass point . . . . . . . . . . . . 571

5.5 Thermodynamics on the lattice . . . . . . . . 576

5.5.1 Thermodynamics for the pure gauge theory . . . . 576

5.5.2 Including dynamical fermions in thermodynamic observables . . . . . . . . . . . . . 581

5.6 Interface tensions . . . . . . . . . . . . . . . . 582

5.6.1 The two-coupling method . . . . . . . . . 584

5.6 .2 The histogram method . . . . . . . . . . . . 585

5.6.3 The vacuum-tunneling correlation length . . . . . . 588

5.6.4 No relics of the type of phase conversion in the early universe? . . . . . . . . . . . . . . 589

5.7 Other lattice actions for QCD and further reading . . . . 590

5.7.1 Simulations with Wilson fermions . . . . . . . . 590

5.7 .2 Improved actions . . . . . . . . . . . . . . 591

5.7.3 QCD at finite baryon density or nonzero chemical potential . . . . . . . . . . . . . . . 592

6. Effective Actions in the Continuum 595

6.1 Postulating effective actions for QCD . . . . . . . . 595

6.2 QCD and dysprosium . . . . . . . . . . 596

6.3 The chiral transition in chiral perturbation theory . . . 602

6.4 Mass sensitivity of the chiral transition . . . . . . . . 605 
6.5 A network of gluonic strings . . . . . . . . . . . . 611

6.6 Further reading . . . . . . . . . . . . . 618

7. Phenomenological Applications to Relativistic Heavy-Ion

Collisions

7.1 The QCD transition in the lab . . . . . . . . . . . . . 619

7.1.1 Scales and observables . . . . . . . . . . . . 619

7.1.2 The hydrodynamic framework . . . . . . . . . 626

7.1.3 The bag-model equation of state . . . . . . . . 631

7.2 Signatures sensitive to the nature of the phase transition . . 635

7.2.1 Thermodynamic observables . . . . . . . . 635

7.2 .2 Dileptons ................. 639

7.2 .3 Strangeness production . . . . . . . . . . . 648

7.2 .4 Pion interferometry ............ 650

7.2.5 Intermittency analysis ........... 652

7.2 .6 Outlook ............... 656

$\begin{array}{lr}\text { References } & 659\end{array}$

$\begin{array}{ll}\text { Index } & 673\end{array}$ 